\title{
A Comprehensive Survey of Palmprint based Biometric Verification System
}

\author{
Mr. Ravindra Gupta ${ }^{1}$, Mrs. Varsha Namdeo ${ }^{2}$, Ms. Rashmi Chourey ${ }^{3}$ \\ Guide, Computer Science and Technology, RKDF Institute of Science and Technology, Bhopal, India ${ }^{1}$ \\ H.O.D, Computer Science and Technology, RKDF Institute of Science and Technology, Bhopal, India ${ }^{2}$ \\ Student, Computer Science and Technology, RKDF Institute of Science and Technology, Bhopal, India ${ }^{3}$
}

\begin{abstract}
As an emerging biometric technology, palmprint recognition technology has been researched widely and developed rapidly, which is well known for its high verification accuracy, low-price capture device, high reliability and user acceptance. During this instance of time, several different glitches related to palmprint recognition have been addressed. Furthermost of the studies has been done in palmprint recognition due to its stability, reliability and exclusivity. Furthermore, it has been used for law enforcement, civil applications and access control applications. Researchers have proposed a variety of palmprint pre-processing, feature extraction and matching approaches. This paper deliberates and review about the number of investigation works introduced to overcome the difficulties confronted in each stage of palm print verification. Our study on palm print recognition focuses on verifying the palm print in different types of schemes involved.
\end{abstract}

Keywords: Palm Print Recognition, biometric authentication system, person verification, Biometrics, security.

\section{INTRODUCTION}

Biometric authentication has been receiving much interest over the past decade with rising demands in automated personal identification. Among many biometric techniques, palmprint recognition is one of the most trustworthy approaches. Palmprint is the inner part of a person's hand. For centuries, the palm line patterns have popularly been believed to be able to predict a person's future. But its uniqueness and capacity for distinguishing individuals has come to fore only recently.

Palmprint is too one of reliable modality since it possesses the more features than that of other modality like the principal lines, minutiae, orientation, singular points etc. Also the palmprint modality is unique for each individual, furthermore it is universal. The palmprint recognition is used in the civil applications, law enforcement \& numerous such applications where access control is important.

Palm has features such as the geometric features, delta point's features, minutiae, principal lines features, ridges $\&$ creases. The principal lines are namely heart line, head line $\&$ the life line. Figure 2 shows the structure of palmprint. The palmprint contains 3 principal lines which divides the palm into three regions: Interdigital, Hypothenar \& Thenar. An Inter digital region lies above Heart line. Thenar lies below the Life line. And the Hypothenar is between Heart \& Life line. From the palmprint principal lines, minutiae, ridges features can be extracted for the identification.

The palmprint recognition techniques have been grouped into two main categories, first approach is based on the low-resolution features \&the second approach is based on the high-resolution features.

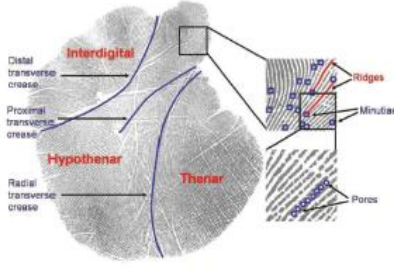

(a)

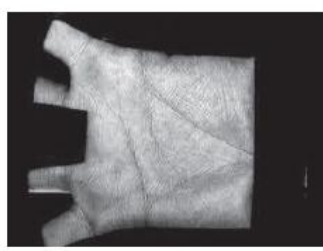

(b)
Fig. 1. Typical palmprint images in different resolution.

(a) High resolution [1]; (b) Low resolution [2]

The first approach makes use of the low-resolution images (like 75 or 150 ppi), where only principal lines, wrinkles, $\&$ the texture are extracted. The second approach uses the high resolution images (like 450 or $500 \mathrm{ppi}$ ), where in the addition to principal lines \& wrinkles, more discriminant features such as ridges, singular points, $\&$ the minutiae can be extracted.

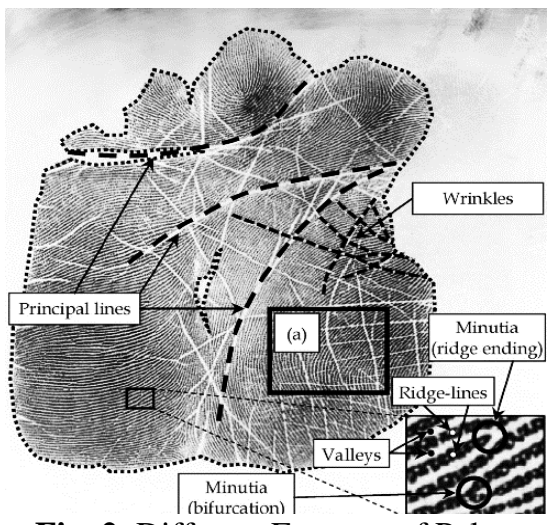

Fig. 2. Different Features of Palm 


\section{A. Palmprint Recognition}

Palmprint, a kind of human physiological trait, has considerable potential for person recognition. It the shares most of discriminative features with the fingerprints, $\&$ in the addition, possesses a much larger skin area \&further discriminative features like the principle lines and creases. Moreover, it is promising to combine palmprints with other hand-based biometrical modalities, such as hand shape, knuckle and palm vein, for achieving a recognition system of higher recognition accuracy and advanced userfriendliness. A typical palmprint recognition system involves four modules, including palmprint image acquisition, image reprocessing, feature extraction, and template comparison. From the view point of palmprint acquisition, the palmprint recognition system can be either offline or online.

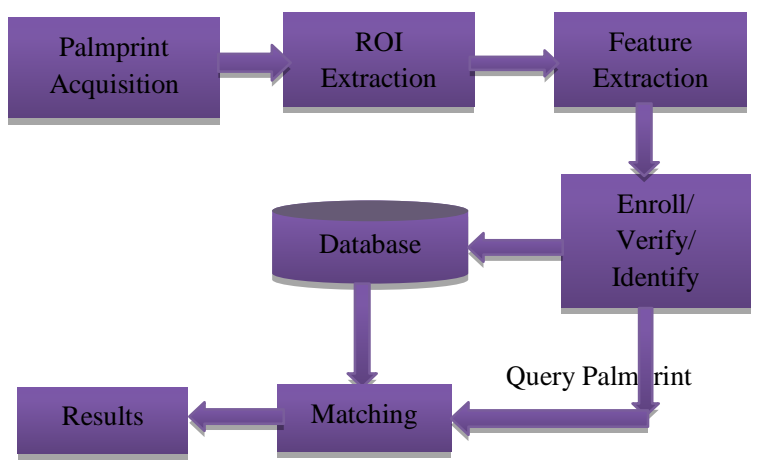

Fig. 3.General block diagram of palmprint recognition system

The offline systems usually work in forensic applications, where palmprint recognition has a significant role since around $30 \%$ percent of the latents recovered from crime scenes are from palms [3]. The online systems are for the civil \& the commercial applications like the access control. The image quality differs significantly for offline and online system. The palmprint images collected from criminal scenes are usually partial and can be digitalized into be high-resolution. Whereas the online palmprint images can be captured from the whole palm but the resolution is generally rather low so as to facilitate realtime processing.

\section{B. Palmprint features}

Figure 1 shows two typical palmprint images in different resolution. In general, there are two basic features in a palmprint: creases and ridges. Both of them are firmly attached to the dermis, and are immutable for the whole life. However, in the palmprint images of different qualities, creases and ridges possess their unique characteristics and therefore play different roles in varieties of applications, which are summarized in Table 1.

The ridges of palm are unique for an individual, just like those in fingerprint. They come into being during the three-to-four months of the fatal stage and are fixed in the adolescence stage [4]. Refer to [3]; ridges can be further divided into ridge pattern, minutia points, ridge contours and pores. Ridge pattern and minutia points can be extracted from palmprint or fingerprint with 500 or less dpi, while ridge contours and pores are from images with resolution of higher than $1000 \mathrm{dpi}$.

TABLE 1 Palmprint features.

\begin{tabular}{|c|c|c|}
\hline \multicolumn{2}{|l|}{ Type } & \multirow{2}{*}{$\begin{array}{l}\text { Characteristics } \\
\text { - } 100 \mathrm{dpi} \\
\text { - Online low-resolution person } \\
\text { recognition }\end{array}$} \\
\hline Creases & $\begin{array}{l}\text { Principal } \\
\text { lines } \\
\text { Wrinkles }\end{array}$ & \\
\hline \multirow[t]{2}{*}{ Ridges } & $\begin{array}{l}\text { Ridge } \\
\text { pattern } \\
\text { Minutia } \\
\text { points }\end{array}$ & \multirow{2}{*}{$\begin{array}{l}\text { - } 500 \text { dpi for ridge patterns and } \\
\text { minutia } \\
\text { - } 1000 \text { dpi for pores and ridge } \\
\text { contours } \\
\text { - Latent palmprint recognition } \\
\text { - Forensics and law } \\
\text { enforcement }\end{array}$} \\
\hline & $\begin{array}{l}\text { Ridge } \\
\text { contours } \\
\text { Pores }\end{array}$ & \\
\hline \multicolumn{2}{|c|}{$\begin{array}{l}\text { Multispectral } \\
\text { features }\end{array}$} & $\begin{array}{l}\text { - Infrared spectral imaging } \\
\text { - Multispectral data fusion for } \\
\text { improving recognition accuracy } \\
\text { - Palm vein information fusion } \\
\text { for improving the capability of } \\
\text { spoof detection }\end{array}$ \\
\hline \multicolumn{2}{|c|}{$\begin{array}{l}\text { 3D structural } \\
\text { information }\end{array}$} & $\begin{array}{l}\text { - 3D imaging } \\
\text { - More robust against fake } \\
\text { palmprint attack } \\
\text { - 2D and 3D information fusion } \\
\text { for a high accuracy and robust } \\
\text { palmprint recognition system }\end{array}$ \\
\hline
\end{tabular}

Ridges features play an important role in the latent palmprint recognition, which has shown great potential in forensics and law enforcement. Figure 1(a) displays a typical palmprint image in forensic applications, where $500 \mathrm{dpi}$ is the standard resolution and latent-to full matching must be supported.

\section{ONLINE PALMPRINT RECOGNITION}

For the online palmprint recognition systems, there are varieties of image acquisition devices. According to the types of sensor, the main devices include digital scanner, CCD (Charge Coupled Device) based palmprint scanner, and web camera. Among them, the web camera is exploited for a touch less system, which usually suffer that the image quality is relatively low. At present, the images in public palmprint databases are basically from digital scanner and CCD based palmprint scanner.

After a palmprint image is captured, some procedures are required to be processed for cropping the region of interest (ROI). With a ROI of equal size extracted from each image, it will reduce the intra-class variations and further facilitate the feature extraction. However, it is a challenge to align the images for ROI cropping. In publications [58], the reader can find some different algorithms of ROI extraction, which usually depend on the image quality and 
the captured palm shape. The general idea involves three steps:

1. Detect key points, whose positions are relatively reliable;

2. Establish a uniform coordinate system based on the detected key points;

3. Crop a region of square shape under the established coordinate system.

Two points around the roots of fingers are detected as the reference points for the coordinate system establishment. In general, the points are finally found out by a sequence of image processing procedures.

However, for an individual, the detected positions of key points vary with samples, due to the image acquisition noise and the palm distortion. In consequence, there are unavoidable variations among the extracted ROI subimages from intra-class palmprint images. In general, the image variations involve rotation, translation and illumination perturbation.

Palmprint feature extraction and matching are processed on the extracted ROI sub-images. Aiming to a palmprint recognition system of high accuracy, a variety of feature extraction and matching approaches have been proposed. Refer to [9], the proposed methods can be grouped into three classes: (1) Holistic-based approaches; (2) Local feature based approaches; (3) Hybrid approaches. By holistic-based approaches, the palmprint images are generally represented either in a spatial domain or a transform domain. The palmprint images can be treated as a vector, a 2D matrix, or a second order tensor and then processed by sorts of linear and nonlinear subspace analysis technologies. Moreover, the palmprints can be firstly transformed by an image transform technique, such as Fourier transforms, discrete cosine transforms (DCT), and Gabor transform, and then processed by subspace analysis technologies. Local feature based approaches mainly resort to detecting and matching the principle lines and wrinkles by some image processing technologies such as image segmentation and enhancement. Hybrid approaches involve the multiple palmprint representations fusion, or some hierarchical matching scheme.

The assessment of this survey paper is as follow; section I contains basic overview and introduction of palmprint recognition system. Online palmprint recognition is described in section II. Complete review of methods for recognition is presented in section III and a conclusion and summary of problem area is presented in section IV.

\section{LITERATURE REVIEW}

\section{Feature-Based Approaches}

In [10] Shu \& Zhang discuss a no. of the features that can be extracted from the palmprint images \& use for the recognition. These features include: (i)geometry features, like the palm width, height $\&$ surface, (ii) the principal lines, (iii) wrinkles, which are smaller \& the less regular than principle lines, (iv) the delta-points, defined as centers of the delta-like regions inside palm, (v) the minutiae, which are alike to features classically an extracted from the fingerprints \& require the highresolution input image to extract.

Figure 4 shows the palmprint with the marked delta-points $\&$ the geometry features. In the offline system, authors used the palm width \& height, as well as the position of numerous points along principal lines as features. On the small database, performing the 48 genuine \& the 844 impostor experiments, they have achieved $0.0 \%$ FAR \& $0.0 \%$ FRR.

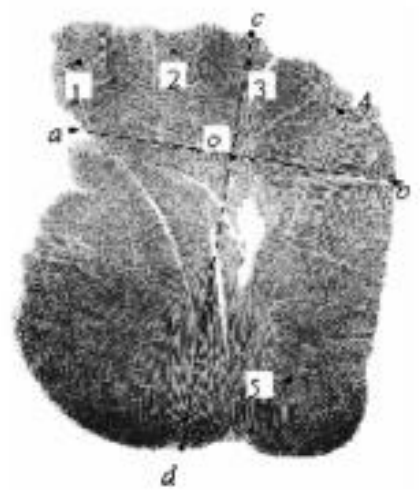

Fig. 4. Palmprint used in [10] withmarked points used to extract the geometry features $(\mathrm{a}, \mathrm{b}, \mathrm{c}, \mathrm{d}) \&$ the deltapoints $(1,2,3,4,5)$.

Zhang \& Shu [11] used the datum points (i.e. points at the end of the principal lines) to align coordinate systems of the different palmprints. The principal line features, obtained using specially crafted filter, were used for the recognition. They tested system on the database of the 20 image pairs of the 20 persons. The ideal result with the $\mathrm{FAR}=0.0 \% \& \mathrm{FRR}=0.0 \%$ was obtained.

Duta et al. [12] used the feature-points obtained as the result of binarization $\&$ the morphological operations (i.e. on their images attained off-line, the principal lines are whiter than rest of the palm). The set of points \& corresponding the orientations was extracted for each palm \& an algorithm was advanced for matching of such attained point sets. An e.g. of matching ofthe point sets is displayed in Figure 4. System was tested on the 30 palmprints of the 3 persons. The mTER of $5.7 \%$ was attained.

You et al. [13] proposed the hierarchical palmprint identification system. In the $1^{\text {st }}$ stage of system, based on the global texture energy, it is examined are the 2 palms similar enough to belong tosimilar person. If they are, system proceeds with the finer matching stage in which the interest points are located on both the palmprints \& matched. An average the recognition rate of $95 \%$ was obtained on database of 200 images of the 100 persons. 


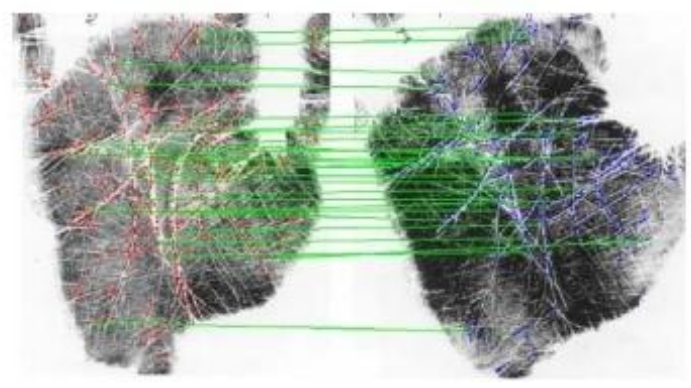

Fig. 5.The palm matching in [12]

Han et al. [14] developed the palmprint authentication system which uses the palmprint images obtained by table scanner. The palmprint features are extracted by using the Sobel edge operator \& the morphological operations on theregion of interest, whose the location is determined based on hand contour $\&$ the position of index-finger. The 2 approaches to matching were experimented with: the template matching \& the back propagation neural networks. In experiment involving 1000 genuine \& the 49000 impostor matching, the FAR \& the FRR below $10 \%$ were obtained with the template-matching approach \& below $2 \%$ by using the neural network.

Zhang et al. [15] developed online palmprint recognition system. The palm images are obtained using the CCD camera. The ring light source is used to guarantee uniform light on surface of palm. After preprocessing \& locating palm region based on the stabile points in valleys between the fingers, system extracts palmprint features using the 2D Gabor filter.

\section{Line-based approaches}

The line-based approaches either develop the edge detectors or use an existing edge detection approaches to extract the palm lines.

Wu et al. designed two masks to compute vertical first-order derivative \& second-order derivative of the palmprint images [16]. Directional first-order \& secondorder derivatives can be attained by rotating two standard marks. They use zero-crossings of first-order derivatives to identify the edge points \& the corresponding directions. The magnitude of the corresponding secondorder derivative is considered as the magnitude of the lines. They retain only the positive magnitude because palm lines are valleys. The weighted sum ofthe local directional magnitude is observed as an element in feature vector. This feature is normalized by its maximum and minimum components. As with [17], Euclidean distance is used for the matching.

Wu et al. propose another algorithm, which use the Sobel masks to compute magnitude of palm lines [18]. These magnitudes are projected along both $\mathrm{x}$ and $\mathrm{y}$ directions to form histograms. These histograms are considered as inputs of Hidden Markov Models (HMMs). Boles et al. use Sobel masks and thresholds to construct binary edge images [19] and then employ Hough transform to extract the parameters of the six lines with highest densities in the accumulator array for matching.
Kung et al. formed the feature vector based on lowresolution edge map. Feature vector is passed into the decision-based neural networks [20]. This was the first paper to report an on-line palmprint recognition method.

Pedro et al. employ Sobel masks to enhance edge information and the statistical information in the processed images is used to estimate an optimal threshold for extracting the edges [21]. The authors then utilize a thinning algorithm to further process the edges. Several descriptors of the edges are computed as features for matching.

Huang et al. proposed 2-level modified finite radon transform \& the dynamic threshold to extract the major wrinkles $\&$ the principal lines. The 2 binary edge maps are compared based on matching scheme called the pixel-toarea comparison [22]. Authors claim that proposed algorithm has a better high false acceptance rate than the classical palmprint identification algorithm -the PalmCode $[23,24]$. However, the PalmCode still has better at the low false acceptance rate. Although some strong wrinkles are included in edge maps, major features in this technique are principal lines, which are genetically dependent [25].

Leung et al. employ Sobel masks to extract palm lines and line segment Hausdorff distance to compare two palmprints [26, 27]. Rafael Diaz et al. use Sobel masks and Morphologic operator as two separated feature extractors to obtain the gradient of the images [28]. These feature values are classified by neural networks.

\section{Gabor}

Gabor filters are also widely used for extracting facial feature vectors. Zhang et al. [29] have used local Gabor binary patterns. They used reduced set of the local histograms based on the Local Gabor Binary Patterns. In proposed method, the face image is first represented by LGBP histograms which are extracted from LGBP images. Then, local LGBP histograms with the high separability \& the low relevance are selected to obtain the dimensionreduced face descriptor.This method gave high reduction in dimensionality and about $94 \%$ accuracy. In [30] Gonzalez \& Castro have proposed another method based on Gabor filter which uses Shape driven Gabor Jets for face description and Authentication.

In [31] Arivazhagan, Mumtaj \& Ganesan Used Multiresolution Transform such as, Gabor Wavelet Transform. The Gabor Wavelet was used to extract spatial frequency, the spatial locality \& the orientation selectivity from the faces irrespective of variations in expressions, illumination $\&$ pose $\&$ then the Normalization was done. Then by seeing each Eigen faces as each the co-ordinate, the coordinate system was formed called the Face space. In this the Face space, each face was considered as point. By projecting each faces, it's the co-ordinate values were determined, which were advanced used for the distance measures in discrimination analysis. The achieved correctness varied from $84-94 \%$ on numerous databases considered. 
Vol. 6, Issue 1, January 2017

\section{Coding Approaches}

Coding approaches encode filter coefficientas the feature using the Gabor filters [32], [33]. Daugman,inventor of the Iris code, has demonstrated that bitwise hamming distance allows the real-time brute force identification in the large databases.

The palm code uses single Gabor filter to extract local phase information of the palm print [34], [35]. D.Zhang et al. usesfirst version of the fusion code to avoid the correlation that results from the palm code. It involves use of 4 directional Gabor filters to createthe 4 palm codes \& these the palm codes are combined [36].

In the second version offusion code, D.Zhang et al.recognized that optimal number of the Gabor filter is 2 . The $2^{\text {nd }}$ version of the fusion code is much more effective than first.

The palm code \& the fusion code employs quantizedphases as features \& hamming distances as matches. The first version ofthe competitive coding scheme uses the multiple two-dimensional Gabor filters to extract theorientation information from the palm lines. This infois then stored in the feature vector called competitive code. Angular distance is used for comparing twocodes [37].

In the second version of the competitive code, 25translated templates are created from an input palm print to match template in the database [38].

Kong et al. introduced the fusion code method to encode phase offilter responses from the bank of Gabor filters with the different orientation. Kong et al. developed competitive code method to encodeorientation information \&achieve state-of-the-art palm print Verification correctness [37].

Wu et al. modified the fusion code to extract orientation field \& uses the hamming distance for thematching [39].Some methods combine numerous imageprocessing approaches to extract the palmprint features \&employ some standard classifiers like the Neural networks to make final decisions [40].

Yue et al. proposed the modified fuzzy c-meanscluster algorithms to definethe orientation of filters. This achieves the higher verification correctness [41]. Zu et al. usethe Probability Feature Image inorder to suppress the random noises in the feature image \& thefuzzy logic was employed in the matching algorithm [42].

Chen et al. [43], [44] perform twodimensionaldual tree complex transform on preprocessed thepalmprints to decompose images. The dual -tree complex transforms are proposed to resolve weakness of the traditional wavelet transform which is not shift invariant, for the pattern recognition. Then they apply the Fourier transform on each sub band \& regard spectrum magnitude as features. Finally, the SVM is used as the classifier.

Hennini-Yeamans et al. employ log -Gabor filters to assign the line content scores to different regions of the palm prints [40]. A Specific number of the regions with the top line -content scores are selected to the train correlation filters. They use the Optimal Tradeoff Synthetic Discriminant Function filter as a classifier.

\section{Subspace based approach}

This method makes use of the Principal Component Analysis (PCA), the Linear Discriminant Analysis (LDA) and the Independent Component Analysis (ICA). The spatial coefficients are considered as the features used for matching. This approach does not need any prior knowledge of the palmprints.

Principal Component Analysis (PCA)

Kim et al. [45] proposed a face recognition method in which they have used the principal component analysis for the dimension reduction and for the feature extraction. In this the two dimensional facial images express into the large one dimensional vector form and main idea of this is to express this one dimensional vector into a compact principal component of feature space. This is also known as eigenspace projection. In this paper they have reshaped the 2-D image of $\mathrm{N} \times \mathrm{N}$ into a $N^{2} \times 1$. And they have taken the ORL database for the simulation and instead of storing all images from the database they have calculated the mean of this databases image and subtract this with all the 1-D $N^{2} \times 1$ images. After subtraction they got the images with unique feature, then they have calculated the eigenvalues and the eigenvectors of the covariance matrix. On the basis of the eigenvalues some eigenvectors are selected as a principal component. After selecting the eigenfaces they have calculated the Euclidean distance. They were considered in the face identification. They have classified the face into the non-face or unknown faces and that the given face is there in the database or not. As all images is highly correlated with itself. And the first eigenface can be used as a filter. The image with low correlation can be rejected or we can say it can be classified into a non-face category. They have selected a threshold on the basis of that they have given the following conclusion:

1. Near face space and near stored face $=\Rightarrow$ known faces.

2. Near face space, but not near a known face $=\Rightarrow$ unknown faces.

3. Distance from face space and near a face class $=\Rightarrow$ non-faces.

4. Distance from face space and not near a known class $=\Rightarrow$ non-faces. [45]

\section{LDA Based Algorithms}

$\mathrm{Lu}$ et al. [46] Low-dimensional characteristic representation with improved discriminatory power is of fundamental importance to biometric recognition frameworks. The greater part of conventional linear discriminant analysis (LDA) based techniques experience the ill effects of the impediment that their optimality criteria are not specifically identified with the classification capability of the acquired characteristic representation. In addition, their arrangement precision is influenced by the "small sample size" (SSS) issue which is regularly experienced in task. They have proposed another 
calculation that arrangement with both of the weaknesses in an efficient and financially effective way.

The proposed here strategy is compared, as far as classification accuracy, to other usually utilized proposed routines on two face databases. Results demonstrate that the execution of the proposed system is general better than those of conventional recognition methodologies, for example, the Eigenfaces, Fisherfaces and D-LDA methods. In this they have used two popular face databases, UMIST and the ORL database to demonstrate the effectiveness of their proposed methods. The ORL database contains 40 subjects with 10 different images per subjects.

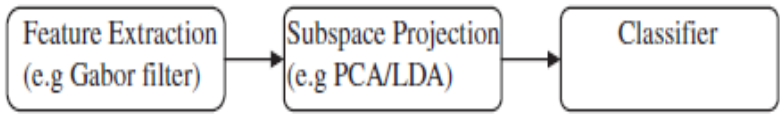

Fig. 6. The architecture of subspace approach

Independent Component Analysis (ICA)

Bartlett and Sejnowski [47] who proposed an ICA representation of face and palm images and compared it with the PCA representation of the same face images. Their study showed that ICA provides a better representation than PCA because in the latter only the second-order statistics are decorrelated. Guan and Szu[48] compared the direct ICA and PCA feature extraction methods for the nearest neighbor classifier for face recognition. Their study found that ICA outperforms PCA when one training image per person is used. It indicates that the direct ICA feature extraction method may be a better alternative when only few training samples are available. Also using the nearest neighbor classifier, Donato et al. [49] showed that ICA representation performed as well as the Gabor representation and better than PCA representation, which are popular representation methods in classifying facial actions.

Kim et al. [50] proposed an ICA based face recognition scheme, which was found to be robust to the illumination and pose variations. An interesting finding by Kim et al. [50] is that in the residual face space ICA provides a more efficient encoding in terms of redundancy reduction than PCA.

In recognition, the algorithms based only on the visual spectrum are not robust enough to be used in uncontrolled environments. Motivated by this question, Chen et al. [51] proposed to fuse information from visual spectrum and infrared imagery to achieve better results. Their scheme also employs ICA as a feature extraction method for the support vector machine (SVM) classifier. Their experimental results show that the scheme improves recognition performance substantially.

Kwak et al. [52] found that ICA transformation can make new features as independent with each other as possible. Similar to earlier studies, the study by Kwak et al. [52] also showed that ICA outperforms PCA and LDA as feature extraction method for face recognition. Subsequently, Kwak and Choi [53] further extended the work by Kwak et al. [52] by developing a stability condition for the earlier study. The two earlier studies mentioned above focused on the two-class recognition problems. More recently, Kwak [54] extended the use of the direct ICA feature extraction method to the case of multi-class face recognition using the nearest neighborhood classifier. The experimental results for several databases demonstrated the usefulness of the direct ICA feature extraction method in solving multi-class face recognition problems.

\section{Multimodal Biometric System}

Yan and Bowyer [55] have proposed biometric recognition using 3D ear shape. In that work, preprocessing of the ear images has had the manual steps \& algorithms had not necessarily the handled problems caused by hair \& earrings. They presented complete system for the ear biometrics, including the automated segmentation of ear in the profile view image $\&$ the 3D shape matching for the recognition. In their system, they achieved rank-one recognition rate of the 97.8 percent for identification scenario \& an equal error rate of the 1.2 percent for the verification scenario on database of the 415 subjects \& 1,386 total probes.

The coding scheme for indexing the multimodal biometric databases was proposed by Gyaourova \& Ross [56]. In the biometric identification systems, identity associated with input data was determined by associating it against every entry in database. That exhaustive matching the process increased response time of system \& potentially, rate of the erroneous identification. A technique that narrows list of the potential identities will allow input data to be matched against the smaller no. of identities. They described a technique for indexing the large-scale multimodal biometric databases based on generation of index code for each enrolled identity. In that the proposed technique, input the biometric data was first matched against the small set of reference images. Set of ensuing match scores was used as index code. Index codes of the multiple modalities were then integrated using three different fusion techniques in order to additional improve indexing performance. Experiments on the chimeric face $\&$ the finger print bimodal database indicated the $76 \%$ reduction in search space at the $100 \%$ hit rate.

Nageshkumar et al. [57] proposed new \& the efficient secure multimodal biometric fusion using the palmprint $\&$ the face image. The biometrics based personal identification was regarded as effective technique for the automatically recognizing, with high confidence a person's identity. In that paper, they have proposed authentication technique for multimodal biometric system identification using 2 traits i.e., face \& palmprint. Proposed system was designed for the application where training data contains a face \& palmprint. Integrating palmprint \& the face features increased robustness of person authentication. Final decision was made by fusion at matching the score level architecture in which the features vectors was created independently for the query 
measures \& was then compared to enrolment template, which was stored during the database preparation.

Zhang et al. [58] proposed thenovel biometric identifier, named the FKP, for the personal identity authentication. First the specific data acquisition device was constructed to capture FKP images \&n then an efficient the FKP recognition algorithm was presented to process acquired data. Local convex direction map of FKP image was extracted based on which the coordinate system was defined to align images \& the Region of Interest (ROI) was cropped for the feature extraction. A competitive coding scheme, which used the 2D Gabor filters to extract image local orientation information, was employed to extract \& represent FKP features. When matching, angular distance was used to measure similarity between 2 competitive code maps. The FKP database was established to examine performance of proposed system \& the experimental results demonstrated efficiency \& the effectiveness of that biometric characteristic.

Zhanga et al. [59] presented thenovel biometric authentication system using the FKP imaging. For matching the 2 FKPs, the feature extraction scheme which combines the orientation $\&$ the magnitude information extracted by the Gabor filtering. The FKP database, which consists of the 7,920 images from the 660 different fingers, has been established to verify efficacy of proposed system \& promising the results were obtained.

Meraoumia et al. [60] proposed novelmethod of the FKP $\&$ the palm print for an efficient the multi-biometric system of the person recognition. The biometric system had been actively emerging in numerous industries for past few years \& that was continuing to roll to offerthe higher security features for the access control system. Addressing that the problem they proposed the efficient matching algorithm based on the PhaseCorrelation Function (PCF) \& using the 2 biometric modalities the palm print \& the FKP. The 2 modalities were combined \& the fusion was applied at matching-score level. Experimental results showed that designed system achieved an excellent recognition rate and provided more security than unimodal biometric-based system.

\section{Touchless Hand Biometrics}

Stenger et al. [61] use a hierarchical model-based approachfor finding and tracking hands. They use a set of models obtained by rotating a basic hand model. The models are organized in a tree structure based on model rotation. Two criteria are used to compare a location on the image to the hand model: edge distance and skin color likelihood. This approach finds the hand shape and orientation but is too slow for real-time applications.

Fratric and Ribaric [62] combine Viola-Jones and a model based approach to locate hand contour in the uncontrolled environments in real-time. Their localization approach first uses Viola-Jones approach to quickly locate suitable hand candidates, and then uses model-based approach, similar to that of Stenger et al. [61] to further verify the candidates and localize the hand. The models used are obtained on a large database of scanned hands.
Because of that, the system can handle moderate variations in hand position. Hand contour and palm region-of-interest are obtained as a result of localization.Figure 6 shows an example of localized hand contour.

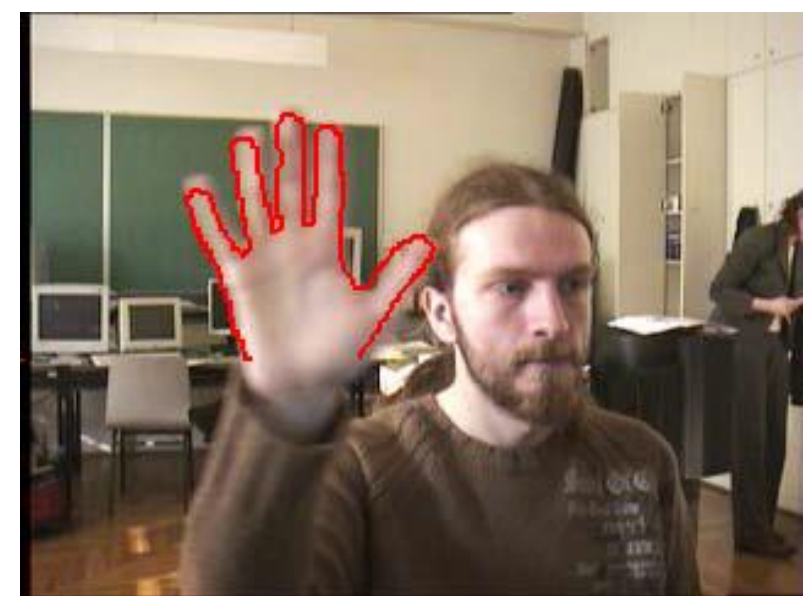

Fig. 7. An example of localized hand contour obtained using [62]

Ong et al. [63] describe a touchless palmprint biometricsystem in which a user is required to place his/her hand above a device, as depicted in Figure 7. Skin color thresholding is used to segment the image and locate the palm region-of interest. Palmprint features are extracted by applying local binary patterns (LBP) on the directional response of the Sobel operator. The drawbacks of the system are, firstly, in using skin color for segmentation, which will not work well in uncontrolled conditions, where skin-colored regions in background can be expected. Secondly, while the system is theoretically able to work in real-time, the authors do not exploit the video information and instead, the system captures a single image every two seconds. The authors report verification EER of $1.52 \%$ on a database of 320 users with 20 images per user.

Doublet et al. [64] also describe a touchless hand recognition system. They also use skin color as a basis for segmentation, but also use active shape model, initialized using the skin color segmentation, to obtain the hand shape.

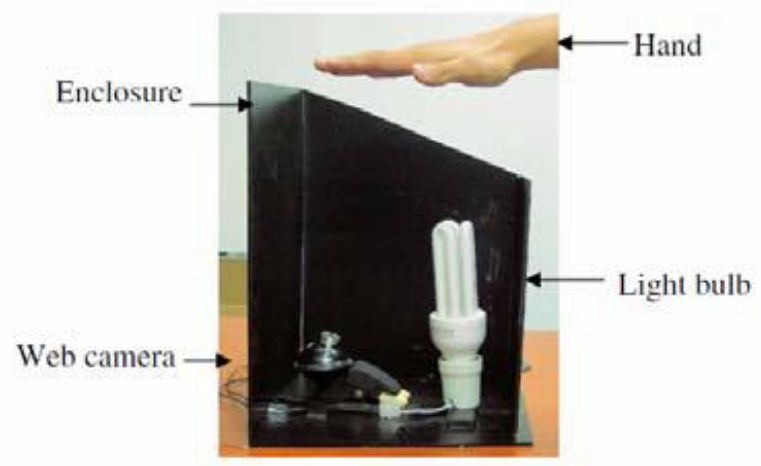

Fig. 8. Touchless device used to capture hand images [touchless6] 
Hand shape is integrated with Gabor-based palmprint representation in order to obtain hand features used in recognition. The whole process of recognition is carried in one second on a $1.6 \mathrm{GHz}$ processor. EER of $1.85 \%$ was reported on a database of 160 images of 16 persons.

Methani and Namboodiri [65] describe a pose correction approach for palmprint recognition in anunconstrained camera based imaging scenario. In their approach, the authors assume the palm is planar and use homography to align two palm images. However, they don't deal with the problem of segmentation at all (the authors use uniform background for experiments), assuming it is a solved problem, and instead deal only with pose changes.

\section{Discrete cosine transform (DCT)\& DWT}

Victor S Viera and Joao Marquez Salomao [66] used Gaussian filter for principle line extraction. Debauchies wavelet transform is applied four times to get features. Nirupama Srinivasanet al., [67] describe an approach to palmprint recognition. A corner finding algorithm is developed to detect the finger tips and trough corners of a palm. Based on this, a consistent region of interest is extracted for each palm. A feature vector is computed for each ROI and similarity index is computed for palms.

Xiang-Qian et al.,[68] describe a palmprint feature called wavelet energy features. This feature is defined employing wavelet, which is powerful tool of multiresolution analysis.WEF can reflect wavelet energy distribution of principal lines, wrinkles, and ridges in several directions at different wavelet decomposition level, so its ability to discriminate palm is very strong.

Vamsi Krishna Madasu [69] describe that fuzzy logic based edge detectors for feature extraction in biometric systems. The edge detection is carried out by means of the local \& the global information. Local information is fuzzified by using the modified Gaussian membership function. Using contrast intensification operator, image is improved to essential level of the visual quality by entropy the optimization of the fuzzification parameters.

Xiangqian Wu et al., [70] described an approach in which principal lines and wrinkles can be extracted in low resolution images. To extract palm a set of directional line detectors are used $\&$ then these detectors are used to remove these lines in the different directions. To avoid losing details of the palm line structure, these an irregular lines are represented using their chain codes. Here, to match the palm lines, the matching score is defined between 2 the palms according to points of the palm lines. Lie Zhang \& David Zhang [71] describe the palmprint identification scheme that characterizes palmprintusing the set of the statistical signatures. Palmprint is the $1^{\text {st }}$ transformed into the wavelet domain, \& the directional context of each the wavelet subband is defined \& computed in order to collect predominant coefficients of it's the principal lines \& wrinkles. The set of statistical signatures, which includes the gravity center, spatial dispersivity, density \& energy, is then defined to characterize palmprints with selected the directional context values.

Yanquing Zhang et al., [72] applied the laplacian transform to palm and middle finger images which are fused together and the feature extraction is done by PCA. Matching is done by Nearest Neighbor Classifier. ShuangXu et al., [73] used Gabor wavelet for feature extraction and represent as a matrix by B2DPCA and further reduced by PCA. RunbinCai et al., [74] fused the visible and infrared images of palmprints. This is then decomposed by DTCWT. The entropy of the fused image and the source image is used for identification. K. Vaidehi, et al., [75] apply DWT DB4 and then DCT for feature extraction. The dimension of DCT is reduced by PCA. Peng -FeiYu et al., [76] used the modified discrete cosine transform based feature extraction technique to obtain the palmprint features. Moreover, the radial basis function the neural network is employed for the palmprint classification. The principal components analysis is applied to decrease these features to reasonable dimension. K B Nagasundara and D S Guru [77] propose a multi algorithm based feature extraction. Features are extracted using both Haar wavelet and Zernike moments. The fused features are then indexed using KD-Tree, which results in faster identification.

\section{Scale invariant feature transform (SIFT)}

The research on multimodal started, and different multimodal biometrics has been developed with combination of various traits, that is, palmprint, face and finger print, face and iris, iris and finger print etc. The most commonly used biometrics is face, that is, either as a single trait or combined with other trait as multimodal biometrics. Face combined with other biometrics at different level of fusion [78], that is, feature, score and decision (Ross and Jain, 2003). Muhammad Imran Razzak, Rubiyah Yusof and Marzuki Khalid presented multimodal face and finger veins biometric authentication [79].

Andreas Uhl and Peter Wild presented multimodal personal verification system using hand images by combining hand geometry and palm image. Directional convolution masks are used to extract the palm futures from normalized palm image, whereas, finger length and width is extracted for hand geometry palm and finally, different level of fusion is executed [80].David G. Lowe presented method for an extracting distinctive invariant features from the images that can be used to perform the reliable matching between the different views of an object or scene [81]. The palm and fingerprint verification was done by applying the Scale invariant feature transform which shows the better results than minutiae points $\&$ the ridge patterns [82]. To determine real potential \& applicability of Scale invariant the feature transform method, the different matching schemes are proposed \& tested using BANCA database \& the protocol, showing better result [83]. 


\section{CONCLUSION \& DISCUSSION}

The increase of terrorism and other sorts of criminal actions, such as fraud in e-commerce, increased the curiosity for more powerful and reliable ways to recognize the identity of a person. To this end, the utilization of behavioural or physiological characteristics, named biometrics, is proposed. Biometrics is best defined as quantifiable physiological and or behavioural traits that can be utilized to verify the distinctiveness of an individual. Many physiological characteristics of humans, i.e., biometrics, are classically invariant over time, easy to acquire, and unique to each individual. Therefore, the biometrics traits are increasingly adopted for civilian use and no longer restricted to forensic identification. The Palmprint is a vital biometric due to the following reasons: 1. Stable structure that is preserved since birth and is quite exclusive to individuals.

2. Invariable to the variation in expression.

3. Immune from anxiety, privacy along with hygiene problems.

Due to the above mentioned causes, automated personal identification using Palmprint images has been increasingly studied for probable commercial applications recently. A typical biometric system usually consists of that precise biometric detection method followed by an extraction methodology (which shrinks the dimensionality of valuable information) and then a classifier to make the suitable decision.

Literature regards two approaches towards palm print recognition - one associated to structural and the other associated to statistical. In the structural approach, the creases, best lines etc. are measured as features and are analysed. On the other hand, some authors determined the datum points derived from principal lines by using the directional projection algorithm. It has been said that, the structural based approaches could extract ridges mostly correctly.

The dominant approach is in the direction of palmprint recognition is based on the statistical features. For statistical based palmprint recognition approach, the works that appear in the literature include Eigen palm (where the original palm print images were projected to a relatively lower dimensional space called Eigen palms), fisher palms (which uses fisher linear discriminant to reduce the dimension), Gabor filters, Fourier Transform, and local texture energy (Out of these approaches, Eigen palm and fisher palm are used for comparing with our approach). We also conclude that the introduction of artificial intelligence in palmprint recognition provide a supervision to all other previous approaches, the methods like neural network, support vector machine, fuzzy logic and other learning paradigm had already increase the efficiency, accuracy and recognition rate of such system. However, it should be noted that appropriate use of such artificial intelligence can increase the recognition rate up to $100 \%$.

\section{REFERENCES}

[1] A. Jain and J. Feng, "Latent Palm print matching," IEEE Trans. Pattern. Anal. Mach. Intell., vol. 31, no. 7, pp. 1032-1047, 2009.

[2] "Biometrics research center (BRC) in HongKong." [Online]. Available: http://www.comp.polyu.edu.hk/_biometrics/.

[3] A. Jain, P. Flynn, and A. Ross, Handbook of Biometrics. Springer, 2007.

[4] F. Galton, Fingerprints. Wm. S. Hein Publishing, 2002

[5] D. Zhang, W. Kong, J. You, and M. Wong, "Online Palmprint Identification," IEEE Trans. Pattern. Anal. Mach. Intell., vol. 25, no. 9, pp. 1041-1050, 2003.

[6] A. Kumar and D. Zhang, "Integrating Shape and Texture for Hand Verification," in International Conference on Image and Graphics, Hong Kong, China, 2004, pp. 222-225.

[7] C. Poon, D. Wong, and H. Shen, "A New Method in Locating and Segmenting Palmprint into Region-of-Interest," in IEEE 17th Int. Conf. Pattern Recognition (ICPR'2004), vol. 4, Cambridge, UK, 2004, pp. 533-536.

[8] Y. Han, Z. Sun, F. Wang, and T. Tan, "Palmprint Recognition under Unconstrained Scenes," in Asian Conference on Computer Vision, vol. LNCS 4844, Tokyo, Japan, 2007, pp. 1-11.

[9] D. Zhang, W. Zuo, and F. Yue, "A Comparative Study of Palmprint Recognition Algorithms," ACM Comput. Surv, vol. 44, no. 1, pp. 2:1-2:37, 2012

[10] W. Shu and D. Zhang, "Automated Personal Identification by Palmprint", Optical Engineering, Vol. 37, pp. 2359-2362, 1998.

[11] D. Zhang and W. Shu, "Two Novel Characteristics in Palmprint Verification: Datum Point Invariance and Line Feature Matching", Pattern Recognition, Vol. 32, pp. 691-702, 1999.

[12] N. Duta, A. K. Jain and K. V. Mardia, "Matching of Palmprints", Pattern Recognition Letters, Vol. 23, pp. 477-485, 2001.

[13] J. You, W. Li and D. Zhang, "Hierarchical Palmprint Identification via Multiple Feature Extraction", Pattern Recognition, Vol. 35, pp. 847-859, 2002.

[14] C. C. Han, H. L. Cheng, K. C. Fan and C. L. Lin "Personal Authentication Using Palm-print Features", Pattern Recognition, Vol. 36, pp. 371-381, 2003.

[15] D. Zhang, W.K. Kong, J. You, and M. Wong, "Online Palm Print Identification", IEEE Trans. Pattern Analysis and Machine Intelligence, Vol. 25, No. 2, pp. 1041-1050, 2003.

[16] X. Wu, K. Wang and D. Zhang, "Line feature extraction and matching in palmprint", in Proceeding of the Second International Conference on Image and Graphics, pp. 583-590, 2002.

[17] X. Wu, K. Wang and D. Zhang, "Fuzzy direction element energy feature (FDEEF) based palmprint identification", in Proceedings of International Conference on Pattern Recognition, vol. 1, pp. 95-98, 2002.

[18] X. Wu, K. Wang and D. Zhang, "HMMs based palmprint identification", Lecture Notes in Computer Science, Springer, vol. 3072, pp. 775-781, 2004.

[19] W.W. Boles and S.Y.T. Chu, "Personal identification using images of the human palms", in Proceedings of IEEE Region 10 Annual Conference, Speech and Image Technologies for Computing and Telecommunications, vol. 1, pp. 295-298, 1997.

[20] S.Y. Kung, S.H. Lin and M. Fang, "A neural network approach to face/palm recognition" in Proceedings of IEEE Workshop Neural Networks for Signal Processing, pp. 323-332, 1995.

[21] P.A Recobos Rodrigues and J.D. Landa Silva, "Biometric identification by dermatoglyphics", in Proceedings of International Conference on Image Processing, vol. 1, pp. 319-322, 1996.

[22] D.S. Huang, W. Jia and D. Zhang, "Palmprint verification based on principal lines", Pattern Recognition, vol. 41, no. 4, pp. 1316-1328, 2008.

[23] W.K. Kong and D. Zhang, "Palmprint texture analysis based on low-resolution images for personal authentication", in Proceedings of 16th International Conference on Pattern Recognition, vol. 3, pp. 807-810, 2002.

[24] D. Zhang, W.K. Kong, J. You and M. Wong, "On-line palmprint identification", IEEE Transactions on Pattern Analysis and Machine Intelligence, vol. 25, no. 9, pp. 1041- 1050, 2003.

[25] A. Kong, D. Zhang and G. Lu, "A study of identical twins palmprint for personal verification", Pattern Recognition, vol. 39, no. 11, pp. 2149-2156, 2006. 


\section{International Journal of Advanced Research in Computer and Communication Engineering} ISO 3297:2007 Certified

Vol. 6, Issue 1, January 2017

[26] M.K.H Leung, A.C.M Fong and H.S. Cheung, "Palmprint verification for controlling access to shared computing resources", IEEE Pervasive Computing, vol. 6, no. 4, pp. 40- 47, 2007

[27] Y. Gao and M.K. Leung, "Face recognition using line edge map", IEEE Transactions on Pattern Analysis and Machine Intelligence, vol. 24, no. 6, pp. 764-779, 2002.

[28] M. Rafael Diaz, C.M. Travieso, J.B. Alonso and M.A. Ferrer, "Biometric system based in the feature of hand palm", in Proceedings of 38th Annual International Carnahan Conference on Security Technology, pp. 136-139, 2004

[29] [Zhang W., Shan S., Chen X, Gao W, "Local Gabor Binary Patterns Based On Mutual Information for Face Recognition", International Journal of Image and Graphics Vol. 7, No. 4 (2007) 777-793, World Scientific.

[30] González D., Alba-Castro J., "Shape-Driven Gabor Jets for Face Description and Authentication", IEEE Transactions on Information Forensics and Security, Vol. 2, No. 4, December 2007, DOI = 10.1109/TIFS.2007.910238

[31] Arivazhagan S, Mumtaj J., Ganesan L., "Face Recognition using Multi-Resolution Transform", International Conference on Computational Intelligence and Multimedia Applications 2007. IEEE DOI 10.1109/ICCIMA.2007.

[32] X. Wu, K. Wang, D. Zhang, and Palmprint authentication based on orientation code matching, in: Proceeding of Fifth International Conference on Audio- and Video based Biometric Person Authentication, 2005, pp. 555-562.

[33] W. Jia, D.S. Huang, D. Zhang, Palmprint verification based on robust line orientation code, Pattern Recognition 41 (5) (2008) 1504-1513.

[34] D.Zhang, Palmprint Authentication, Norwell, mass. Kluwer Academic publishers, 2004

[35] D. Zhang, W.K. Kong, J. You, M. Wong, On-line palmprint identification, IEEE Transactions on Pattern Analysis and Machine Intelligence 25 (9) (2003) 1041-1050.

[36] Q. Li, Z. Qiu, D. Sun, Feature-level fusion of hand biometrics for personal verification based on Kernel PCA, International Conference on Biometrics, 2006, pp. 744-750.

[37] A.W.K. Kong, D. Zhang, Competitive coding scheme for Palm print verification in: proceedings of international conference on Pattern Recognition, vol.1.2004, pp520-523

[38] A. Kong, D. Zhang, G. Lu, A study of identical twins palmprint for personal verification, Pattern Recognition 39 (11) (2006) 21492156.

[39] X. Wu, D. Zhang, K. Wang, Fusion of phase and orientation information for palmprint authentication, Pattern Analysis and Applications 9 (2) (2006) 103-111.

[40] P. Hennings, B.V.K.V. Kumar, Palmprint recognition using correlation filter classifiers, in: Conference Record of the 38th Asilomar Conference on Signal, Systems and Computers, vol. 1, 2004, pp. 567-571.

[41] X. Wu, K. Wang, D. Zhang, Fuzzy direction element energy feature (FDEEF) based palmprint identification, in: Proceedings of International Conference on Pattern Recognition, vol. 1, 2002, pp. 95-98.

[42] T. Connie, A.T.B. Jin, M.G.K. Ong, D.N.C. Ling, An automated palmprint recognition system, Image and Vision Computing 23 (5) (2005) 501-515.

[43] J.S. Chen, Y.S. Moon, H.W. Yeung, Palmprint authentication using time series, in: Proceeding of Fifth International Conference on Audio- and Video based Biometric Person Authentication, 2005, pp. 20-22.

[44] G.Y. chen, T.D.Bui, a.krzyak, Palmprint classification using dual tree complex wavelets, in: proceedings of international conference on image Processing, 2006, pp 2645-2648.

[45] Kyungnam Kim. Face recognition using principle component analysis. In International Conference on Computer Vision and Pattern Recognition, pages 586-591, 1996.

[46] Juwei $\mathrm{Lu}$, Konstantinos $\mathrm{N}$ Plataniotis, and Anastasios $\mathrm{N}$ Venetsanopoulos. Face recognition using LDA-based algorithms. Neural Networks, IEEE Transactions on, 14(1):195-200, 2003.

[47] Bartlett, M.S., Sejnowski, T.J., 1997. Independent components of face images: A representation for face recognition. In: Proceedings of the 4th Annual Joint Symposium on Neural Computation, Pasadena, CA.
[48] Guan, A.X. Szu, H.H., 1999. A local face statistics recognition methodology beyond ICA and/or PCA. In: Proceedings of the International Joint Conference on Neural Networks (IJCNN'99), Vol. 2, and pp. 1016-1021.

[49] Donato, G., Bartlett, M., Hager, J., Ekman, P., Sejnowski, T., 1999. Classifying facial actions. IEEE Transactions on Pattern Analysis and Machine Intelligence 21, 974-989.

[50] Kim, K.J., Cho, S.B., 2004. Prediction of colon cancer using an evolutionary neural network. Neurocomputing 61, 361-379.

[51] Chen, X., Jing, Z., Xiao, G., 2007. Nonlinear fusion for face recognition using fuzzy integral. Communications in Nonlinear Science and Numerical Simulation 12, 823-831.

[52] Kwak, N., Choi, C., Ahuja, N., 2002. Face recognition using feature extraction based on independent component analysis. In: Proceedings of International Conference on Image Processing, pp. 337-340.

[53] Kwah, N., Choi, C., 2003. Feature extraction based on ICA for binary classification problems. IEEE Transactions on Knowledge and Data Engineering 15, 1374- 1388

[54] Kwak, N., 2008. Feature extraction for classification problems and its application to face recognition. Pattern Recognition 41, 17011717

[55] Yan P. and Bowyer K., "Biometric Recognition using 3D Ear Shape," Pattern Analysis and Machine Intelligence, vol. 29, no. 9, pp. $1297-1308,2008$.

[56] Gyaourova A. and Ross A., "A Coding Scheme for Indexing Multimodal Biometric Databases," in Proceedings of IEEE Computer Society Conference on Computer Vision and Pattern Recognition Workshops, Florida, USA, pp. 93- 98, 2009.

[57] Nageshkumar M., Mahesh P., and Swamy M., "An Efficient Secure Multimodal Biometric Fusion using Palmprint and Face Image," the International Journal of Computer Science Issue, vol. 1, pp. 49-53, 2009.

[58] Zhang L., Zhang L., and Zhang D., "Finger Knuckle-Print: A New Biometric Identifier," in Proceedings of the 16th IEEE International Conference on Image Processing, Cario, Egypt, pp. 1981-1984, 2009

[59] Zhang L., Zhang L. and Zhang D., and Zhu H., "Online FingerKnuckle-Print Verification for Personal Authentication," the Journal of Pattern Recognition, vol. 43, no. 7, pp. 2560-2571, 2010.

60] Meraoumia A., Chitroub S., and Bouridane A., "Fusion of FingerKnuckle-Print and palm print for an Efficient Multi-Biometric System of Person Recognition," in Proceedings of the IEEE International Conference on Communications, Kyoto, Japan, pp. 1$5,2011$.

61] B. Stenger, A. Thayananthan, P.H.S. Torr and R. Cipolla, "Modelbased hand tracking using a hierarchical Bayesian filter" IEEE Trans. Pattern Analysis and Machine Intelligence, Vol. 28, No. 9, pp. 1372-1384, 2006

[62] I. Fratric and S. Ribaric, "Real-Time Model-Based Hand Localization for Unsupervised Palmar Image Acquisition", Proc. Int'l Conference on Biometrics 2009, pp. 1280-1289.

[63] M.G.K. Ong, T. Connie and A.B.J. Teoh, "Touch-less palm print biometrics: Novel design and implementation", Image Vision Computing, Vol. 26, No. 12, pp. 1551-1560, 2008

[64] J. Doublet, O. Lepetit and M. Revenu, "Contact-less Hand Recognition using shape and texture features", 8th Int'l Conference on Signal Processing, Guilin, China, November, 2006.

[65] C. Methani and A.M. Namboodiri, "Pose Invariant Palmprint Recognition", Proc. Int'l Conference on Biometrics 2009, pp. 577-586.

[66] Vieira V S and Salomao J M, "Use of Wavelet Transforms and Neural Networks for Identifying Individuals through Extracted Features of the Palm Hand." International Conference on Biosignals and Bio-robotics Conference, pp. 1-5, 2011

67] Nirupama Srinivasan and Evangelia Micheli-Tzanakou, "Palmprint Recognition: A New Algorithm For Corner Detection Using Palm Anatomy Features", IEEE International Workshop on Measurement Systems for Homeland Security, Conraband Detection and personal Safety, pp 6-9,2006.

[68] Xiang-Qian Wu, Kuan-Quan Wang, and David Zhang, "Wavelet Based Palmprint Recognition", Proceedings of First International Conference on Machine Learning and Cybernetics, 2002. 
[69] Vamsi Krishna Madasu, Shantaram Vasikarla, "Fuzzy Edge Detection in Biometric Systems", 36th Applied Imagery Pattern Recognition Workshop, 2007.

[70] Xiangqian Wu, David Zhang, and Kuanquan Wang, "Palm Line Extraction and Matching for Personal Authentication", IEEE Transactions on Systems, Man and Cybernetics-Part A: Systems and Humans, vol. 36, no.5, 2006.

[71] Lei Zhang and David Zhang, "Characterization of Palmprints by Wavelet Signatures via Directional Context Modeling", IEEE Transactions on systems, Man and Cybernetics, vol. 34, no.3, 2004.

[72] Yanqiang Zhang, Dongmei Sun and Zheng ding Qiu, "Hand-Based Feature Level Fusion for Single Sample Biometrics Recognition", Emerging Techniques and Challenges for Hand-Based Biometrics, Istanbul, pp 1 - 4, August 2010.

[73] ShuangXu, JidongSuo, JiYin Zhao and Jifeng Ding, "A BiDirectional Compressed 2DPCA for Palmprint recognition based on Gabor wavelets". Sixth International Conference on Natural Computation, pp 958-961, 2010.

[74] Runbin Cai and Dewen Hu, "Image fusion of palmprint and palm vein: Multispectral palm image fusion", IEEE International Congress on Image and Signal Processing, Vol. 6, pp 2778 - 2781, Oct 2010.

[75] K. Vaidehi, T S Subhashini, V Ramalingam, S Palanivel and M Kalaimamani, "Transform based Approaches for Palmprint Identification", International Journal of Computer Applications, Vol 41, pp 1-5, 2012.

[76] Peng Fei-Yu and Dan Xu, "Palmprint recognition based on modified DCT features and RBF neural network, International Conference on Machine Learning and Cybernetics, pp. $2982-$ 2986, July 2008.

[77] K B Nagasundara and D S Guru, "Multi Algorithm based Feature Palmprint Indexing" International Journal of Computer Applications, pp 7-12, 2012.

[78] A. Ross and A. Jain, Information Fusion in Biometrics, Pattern Recognition Letters 24 (2003), pp. 2115-2125.

[79] Multimodal face and finger veins biometric authentication Muhammad Imran Razzak1, 2*, Rubiyah Yusof1 and Marzuki Khalid Center of Artificial Intelligence and Robotics, Universiti Teknologi, Malaysia.

[80] Image Fusion using Complex Wavelets Paul Hill, Nishan Canagarajah and Dave Bull Dept. of Electrical and Electronic Engineering the University of Bristol.

[81] D. G. Lowe, "Distinctive image features from scale invariant Keypoints", International Journal of Computer Vision, vol. 60, no. $2,2004$.

[82] U. Park, S. Pankanti and A. K. Jain, "Fingerprint Verification Using SIFT Features", Proceedings of SPIE Defense and Security Symposium, Orlando, Florida, 2008.

[83] Integrating Faces and Fingerprints for Personal Identification Lin Hong and Anil Jain, Fellow, IEEE. 